\title{
Ex-situ Technology Appropriation of an e-Deliberation Platform in an Art Gallery
}

\author{
Anna Maria Al Zubaidi-Polli ${ }^{1}$, Nervo Verdezoto ${ }^{2}$, Nawfal Al Zubaidi R-Smith ${ }^{3}$ \\ Gabriele Anderst-Kotsis ${ }^{1}$ \\ ${ }^{1}$ Department of Telecooperation, Johannes Kepler University Linz, Altenbergerstrasse 69, 4040 Linz, Austria \\ ${ }^{2}$ Department of Informatics, University of Leicester, University Road, LE1 7RH Leicester, United Kingdom \\ ${ }^{3}$ Department of Radio Electronics, Brno University of Technology, Technicka 12, 61600 Brno, Czech Republic \\ anna.polli@fh-hagenberg.at
}

\begin{abstract}
When designing interactive web applications and services, understanding the situated nature of interaction and investigating technology appropriation experiences in the context of real-world use deserve special attention. In this paper, we report on experiences with technology appropriation of an e-Deliberation platform that we designed to support collaborative interpretation in an art gallery. Our qualitative study is based on interviews with 23 participants to explore a) visitors' practices when commenting on and interpreting art and b) how these practices are shaped by interaction with an e-Deliberation platform for collaborative writing that imposes strict regulations. An analysis of 12 hours of interview data yielded two particular appropriation practices related to the demand to satisfy additional visitors' communication needs, such as exchanging their ideas and thoughts face-to-face before writing an interpretation. Although the designers anticipated these specific needs, users also appropriated the e-Deliberation platform in unanticipated ways. These practices lead to a better understanding of the situated nature of ex-situ interaction when using interactive web applications to support remote collaboration in the art gallery context.
\end{abstract}

\section{CCS Concepts}

- Human-centered computing $\rightarrow$ Human computer interaction (HCI)

\section{Keywords}

Appropriation practices, E-Deliberation platform, Initial familiarity, Interpretation writing, Communication needs

Permission to make digital or hard copies of all or part of this work for personal or classroom use is granted without fee provided that copies are not made or distributed for profit or commercial advantage and that copies bear this notice and the full citation on the first page. Copyrights for components of this work owned by others than the author(s) must be honored. Abstracting with credit is permitted. To copy otherwise, or republish, to post on servers or to redistribute to lists, requires prior specific permission and/or a fee. Request permissions from Permissions@acm.org.

iiWAS '17, December 4-6, 2017, Salzburg, Austria (C) 2017 Copyright is held by the owner/author(s). Publication rights licensed to ACM.

ACM ISBN 978-1-4503-5299-4/17/12 ..\$15.00

https://doi.org/10.1145/3151759.3151761

\section{INTRODUCTION}

The concept of appropriation has engaged several research communities over the last 15 years. Research has broadened our understanding of how people adopt and adapt technologies [1] as well as how people fit them into the routines of their everyday lives $[2,3,4,5,6]$. Appropriation may occur because existing features of a specific technology are designed to support it or when a system does not meet the needs of the intended user, who may then have to re-adapt it. In fact, people domesticate technology and use it in their own ways by adding and applying further physical and digital tools with which they are familiar from their everyday practices to ease interaction [3]. When this has occurred, a technology has been appropriated and has become the user's own and is no longer simply what we - as designers provided. Such improvisations and adaptations around technology are not a sign of failure [7]; rather, they show how technology can be domesticated when users understand and feel comfortable enough to use technology in their own ways [3]. When technology is flexibly designed, it can easily be shaped by people [8], and they may feel more in control of a collaborative activity and thereby establish a sense of ownership [9] through its use and appropriation.

A good example of technology that has been domesticated is Wikipedia, an e-Service platform that supports collaborative writing. The web-based, free-content encyclopedia project, Wikipedia has been a role model due to its flexibility in enabling constructive participation in online collaborative deliberation [9]. An e-Service can be available not only at any time, but also anywhere [10]. However, availability is not the only advantage; further successful factors and methods of e-Applications have been identified that have an impact on sustainability [11] and therefore provide permanent access. Giving people continuous access to a web-based format of contributions - as in Wikipedia, where both novices and Wikipedians are able to contribute to articles, no matter where they are located - can increase their feeling of being part of the production of the collaborative text rather than being mere spectators [9]. At the iiWAS2015 conference, Johansson et al. [10] presented various concepts for enhancing citizen participation and cooperation, and highlighted the importance of investigating the adoption of e-Services that can transform citizen involvement.

In this study, we explored such an approach, investigating whether people gain a sense of ownership by participating in writing one collaborative interpretation. We invited 23 participants to use an e-Deliberation platform collaboratively to 


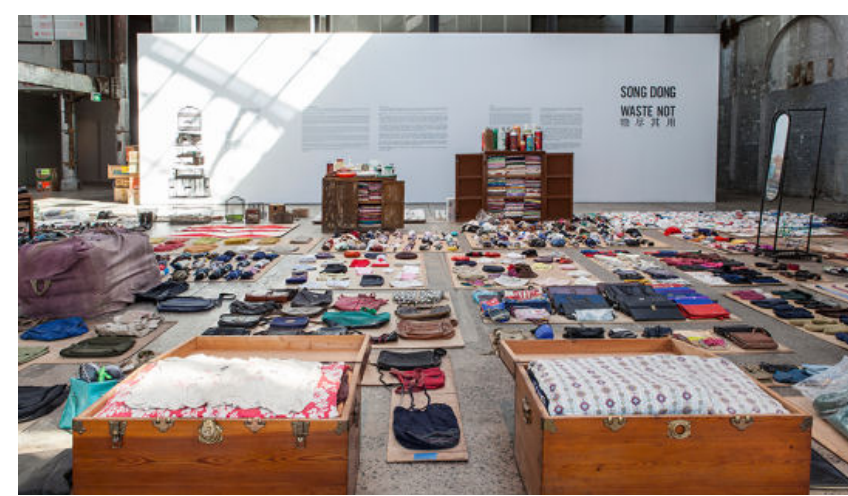

Figure 1: Overview of the setting - The artwork and the interpretation panel (on the white wall) before the replacement with our e-Deliberation platform. (C) Vin Rathod

write an interpretation of a selected artwork in a gallery in Aarhus, Denmark. We conducted a design experiment in which the traditional text description panel next to the selected artwork, entitled "Waste Not" by the artist Song Dong (Figure 1), was replaced with our e-Deliberation platform technology, which allowed visitors to use and share an online space for collaborative interpretation writing.

The visitors had access to the platform both in-situ (when close to the selected artwork) and ex-situ (when remote from the artwork) [12]. Our findings describe two examples of technology appropriation where existing features of an open-source eDeliberation platform (customized for use in this study) were appropriated in unexpected ways.

In the following sections, we present related work, describe our research approach, and identify the technology appropriation practices based on our preliminary analysis. We then present how these practices relate to the communication needs in the context of the art gallery and their implications for future design of eDeliberation tools and services.

\section{RELATED WORK}

The Human Computer Interaction (HCI), Information Integration and Web-based Applications \& Services (iiWAS) and ComputerSupported Cooperative Work and Social Computing (CSCW) communities have long been concerned with understanding and supporting technology-mediated collaboration between various actors in society $[13,14,15]$. Use of technology is becoming pervasive in everyday life, and the scope of user practices and collaboration has broadened from work [1] to everyday settings $[4,5]$. The HCI, iiWAS and CSCW communities talk about technology that can be appropriated and shared by everybody and integrated into people's everyday lives. Semi-public spaces, such as museums, have demonstrated how technological support can make visitors' experiences of exhibitions more interactive and engaging [16, 17]. However researchers have encountered several challenges, such as designing flexible installations [8] that people can easily appropriate, providing users with a sense of ownership [3] and the opportunity to leave a trace behind them when leaving the physical place [16], and designing such that users can be part of a collaborative knowledge production [9].

Researchers propose investigating the process of appropriation within practices not only from a theoretical perspective (e.g., [18, 19]), but also considering bottom-up observational approaches (e.g., [20]). For instance, Dourish [1] looked at appropriation from a sociotechnical perspective, considering a bottom-up approach and describing appropriation as the co-evolution between the practices of the technology and its deployment. Dourish investigated users' working practices and the features facilitating technology appropriation through the use of a document management system deployed in an organizational environment. Dourish developed design principles to help designers in developing open and flexible technologies [1]. When these design principles are followed, they support the social meanings created around the technologies and provide opportunities for their appropriation by a variety of users. Dourish described appropriation as a process by which people adopt and adapt technologies to better address their needs, discovering new opportunities and inventing new uses not envisioned by the designer [1]. Dourish [1] affirmed that the designer does not predetermine how a technology is used, and that appropriation emerges from the situated activities in which people use the artifact. He claimed that appropriation is key when it comes to the development of interactive systems, as it illustrates the success of the technological deployment. This highlights the importance of appropriation, as it lies at the intersection of design and context of use [1].

In the course of our own research, we came to understand that in restricted contexts such as our case of an e-Deliberation platform, which is characterized by strict regulations, technology appropriation happens for various reasons that were not anticipated, that is, due to unfulfilled communication needs [4]. An informed basis [21] for how to design for the 'unexpected' [3] is therefore crucial when it comes to appropriation practices [4] in order to be able to understand and conceptualize these application contexts [22] .

This paper reflects upon user experiences of appropriating our web-based technology. We studied both successful (i.e., users satisfied their needs) and unsuccessful appropriation (i.e., rejection of the technology) [23], and, provide examples of how technology did not meet particular users' needs. As described by Dourish [1], interrelating such technology appropriation experiences with the actual context of use is of utmost importance for understanding the situated nature of interaction [24] and forms the basis for establishing design guidelines.

\section{THE STUDY}

The project from which the examples of technology appropriation presented in this paper are derived from is entitled Aarhus's Artwork. The aim of this study was to engage people in collaboratively writing an interpretation of a specific artwork. People with a particular interest in art (either due to their specialization or academic background) were invited to participate and write interpretations of one selected piece of art. By bringing into an art gallery an open-source e-Deliberation platform that provides dedicated online tools for online participation and deliberation, we also explored ways of addressing the communication needs of visitors with regard to artworks. Visitors' participation in composing a text about a piece of art that is then presented in the gallery provided us with interesting opportunities to understand how people can engage and leave a trace behind them. Thus how they potentially gained a sense of ownership of the interpretation, the piece of art, the gallery and the technology. Facilitating these written interpretations can support the communication between visitors when ex-situ, that is, distant or remote from the object under discussion - the piece of art. 
Our study involved 23 participants from the Department of Aesthetics and Communication at Aarhus University with shared interests in discussing art and curatorial writing. The group of participants consisted of 19 female and 4 male students, aged between twenty-two and thirty-six, with a mean age of twenty-six years. More than half of the participants lived in Aarhus, the city where the art gallery is located. The others commuted daily by public transport from nearby cities to university. We chose the participants based on their interest in interpreting art and visiting museums. A further reason for inviting these 23 students to participate in our study was their field of academic study related to art. Our study was conducted alongside a master's course the students were attending for a quarter-semester that addressed techniques and ways to interpret art. The students volunteered to participate in our study. This specific course provided us with the possibility to meet all students simultaneously throughout the study. The students were invited to use our e-Deliberation platform, which was deployed for a three-month period, to collaboratively write their interpretations. We were interested in understanding how the collaborative interpretation of a specific piece of art in a gallery evolves over time.

This project was a follow-up study to the Local Area Artwork project $[4,17,25]$. In our previous study, we found that visitors to the art gallery wrote scattered comments about the artworks rather than - as we had anticipated - an interpretation or coherent text. The users appropriated the technology, fitting it to their needs to facilitate their use of it [4, 17]. Further, the participants in this initial study used the technology only with in-situ access (when close to the artwork under discussion). Some of the visitors interviewed indicated that they would have needed more time to reflect upon the piece of art in order to be able to write a coherent interpretation rather than a short comment.

The follow-up study reported here used an e-Deliberation platform, which enabled access from anywhere (i.e., in-situ and ex-situ).

\section{THE OPENDCN PLATFORM}

We used a bespoke open-source e-Deliberation platform developed jointly by the Foundation RCM (FRCM) and the Civic Informatics Laboratory (LIC) [26] that supports decision-making and helps to finalize discussions to arrive at a consensus among participants. This e-Deliberation platform, called openDCN (DCN meaning Deliberative Community Networks), is a socio-technical computer-enabled trusted environment for e-Deliberation. It was designed for deliberative purposes that are based on democratic principles and provides dedicated online tools for supporting online participation.

\subsection{One deliberative space and its four tools}

The openDCN comprises four deliberative tools that support multiple participation modalities, each of which has its own participatory feature designed to meet various needs. The first tool is called deliberative brainstorming, which fosters the selection of ideas and proposals and their refinement. The second is $e$ Consultation, which polls the opinions of a relevant number of people who choose between established possibilities. Online deliberation, the third tool, is used for a structured debate that is regulated by a pre-established protocol. The last deliberative tool is the group-decision feature, which supports group decisions with mathematical algorithms for choosing a proposal of interpretations within a set of alternatives under consideration [27]. These tools support various participation modalities, and not all of them necessarily belong to just one space. For example, the openDCN citizen-consultation tool, typically used in deliberation, can also be set up to survey participants' ideas with the aim of using the results to spur debate or to foster a sense of community [26].

\subsection{Adaptation of the openDCN platform}

The web-based openDCN platform builds upon open-source technologies only. The platform was implemented mainly in PHP using the CAKEPHP development framework and the Smarty template engine. For additional functionalities, the Content Management Platform Drupal (http://drupal.org) was used. For our openDCN platform, we customized around $40 \%$ of Drupal's original functionalities relating to uploading, preferences, blog, forums and posting comments. The Certified Consultation was developed in Java [28].

\subsubsection{The concept of using the human-based genetic algorithm to find a contextual consensus}

For choosing the best interpretation proposal within a set of potential alternative solutions, we applied a human-based genetic algorithm (HBGA) [26, 28, 29, 30] [31]. The best solution is found through approximations. Each solution is tested at a small cost. These tests provide feedback to the algorithm on how well each solution satisfies the requirements it is trying to fulfill. It is the human that provides the algorithm with the assessment of a solution's fitness. Given a set of solutions by the human, it provides alternatives. Solutions with low fitness score poorly and are therefore discarded. Solutions with high fitness are considered to be good solutions and are therefore highlighted and retained. These highlighted solutions can be modified in the next iteration cycle to generate new possible solutions. A semi-continuous interaction between the participants and the e-Deliberation platform is needed in order to obtain the best solution. First, the platform asks each participant to give a possible solution. Once the solutions have been collected, they are presented back to the participants, who must then rank each with $0-3$ stars. This evaluation is then used by the e-Deliberation platform to assess the fitness of each existing solution and to decide which solutions are to be discarded and which are kept and highlighted. The surviving solutions are then fed back to the participants, with the instruction to produce new solutions by mutating those presented [27]. In our case, the algorithm allowed the participants to tackle open questions and to collaboratively draft an art interpretation by having each of them propose one interpretation and then evaluate those of the others.

The platform had been tested previously in the context of supporting public discussions of the spring 2006 municipal election in Milan (Italy). There, De Cindio et al. [27] surveyed participants' ideas with the aim of using the results to spur debate and to foster a sense of community. The openDCN platform can be downloaded from SourceForge ${ }^{1}$. The Italian political party Movimento 5 Stelle and the Italian political mass protest movement of II Popolo Viola also used this platform [27].

\subsection{Deployment of openDCN in our research}

We modified this open-source platform by adding constraints to better fit our research focus on discussing art and collaboratively writing interpretations. First, we constrained the exact contents of an interpretation: name of the artist, location, title of the artwork, year of creation and a description of the artwork (i.e., inspiration

\footnotetext{
${ }^{1}$ https://sourceforge.net/projects/openden/
} 
to create the piece, techniques used for creation, what it represents and what it means to the interpreter). Second, we set up a timetable and a process to be followed over the three-month period of deployment, where each participant was initially invited to submit their individual written interpretation. Participants were given a week within which to submit their interpretations; these were then published on the e-Deliberation platform, where all participants could read them. After reading each other's personal interpretations, the participants were asked to evaluate each interpretation, ranking them from 0-3 stars. Next, the HBGA evaluated the participants' solutions. Once this evaluation phase had been completed, the results of the collective ranking were published, and the initial interpretations were labeled as discarded or highlighted based on their rankings. In the next phase, the participants were invited to write new interpretations based on the highlighted ideas. Here, the collaborative aspect already came into play in the first iterative cycle. This process was repeated four times, yielding 4 complete iterations, until consensus on one text was reached. The structure of this process and the length of each phase were constrained to arrive at one consensus interpretation. Over this process of writing, communicating and rewriting interpretations, characteristics of technology appropriation were identified in the preliminarily analysis of the study. Although the specific context of use imposed some constraints and the process of writing or rewriting an interpretation contained some predefined steps, appropriation proceeded unhindered.

\section{PROCESS OF COLLABORATIVE WRITING USING THE E-DELIBERATION PLATFORM}

In an introductory session with the participants, the project timeline and the process of the study were discussed and arranged. After creating personal accounts on the DCN platform, the participants were able to join the collaborative interpretation writing. Through discussions with the students' lecturer, we predefined the process to comprise four iterations. One cycle consisted of three simple steps: 1) writing an interpretation individually and submitting it to the platform, 2) reading of and voting on all interpretations submitted, and 3) re-writing the interpretation based on the highest-scored interpretations. Four of these cycles took place over a three-month period. The users received regular e-mail notifications regarding each change of phase. An evaluation of active and passive participation throughout the four iterations will be presented in a future publication focusing on our quantitative data [32].

\subsection{Step 1: Collection of interpretations (Proposal Phase)}

As illustrated in Figure 2 (left side), using the openDCN platform, the participants were first given one week to submit their interpretations of the chosen artwork. Participants could not view each other's submissions from the current iteration. Once the deadline had been reached, the system administrator (the first author of this paper) advanced the process to the next phase - the Evaluation Phase - and all interpretations submitted became visible to all participants, thus completing the first step.

\subsection{Step 2: Voting on all interpretations (Evaluation Phase)}

Participants were invited to vote on and assign a score to each interpretation including their own (see Figure 2 - middle). The higher the number of stars, the more in favor of the interpretation a participant was based on the criteria of style of writing, how convincing the interpretation was, and how suitable it was for placement next to the piece of art in the gallery. The rating system was based on stars. Because of the high number of participants, we reduced the range of the voting system to 0 to 3 stars ( 3 stars being the best rating), thus decreasing the variability and the likelihood of retaining dominated poor or high ideas. In the other e-Deliberation projects the rating range was from 0 to 5 stars [33]. When a participant wished an interpretation to be retained in the final interpretation, 3 stars were awarded. The evaluation phase had a deadline by which the participants had to submit their evaluations. Upon advancing to the next phase - the Calculation Phase - the participants were able to see the results of the voting, and the next iteration started.

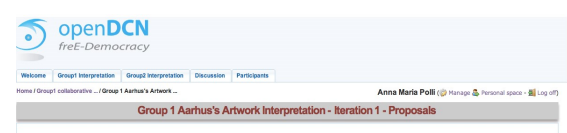

Do you have an interpretation about this artwork? Suggest it $\Rightarrow$

Ideas for this artwork?

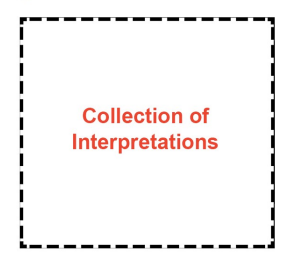

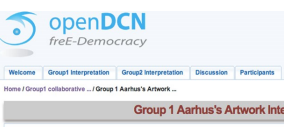

The scenario isn't in the proposal gathering phase. Wait for the end of this iteration to submit your ideas. Iteration 1

Waste Not created by Song Dong is an inspiring work of art which first of all illustrates the life of Song Dong's mother, Zhao Xiangyuan, and her way of overcoming tragic and

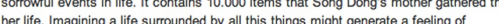
claustrophobia. However, everything which is part of this artwork is categorized organized with extreme precision, and due to this a systematic order appears and an atmosphere of peace and calmness occur. This overwhelming but at the same time peaceful picture furthermore shows the spint of a generation whose believes were based on the idea that nating could evim go to upon modesty and scarcity simultaneously suggests a sense of irresponsibility in the
growing consumerism of present time and in that way indirectly questions our presert growing consumerism of prese
relationship to material things.

Written the 2014-02-21 at 16:39 by Frida Gudmundsson Do you like this idea?

Time - hours, minutes, seconds... Sometimes time flies by, and sometimes it feels so slow. We get older every second, and so does our things. "Waste not" is an exhibition with an overwhelming amount of old used things. When you look at these things, time becomes very near - you can literally see the time that has past in these things. In time stories are told. Happy, sad and tragic ones. These stories live on in the things we posses. What stoy those your things tell? What is your story? And in time, how will people respond to your

Written the 2014-02-21 at 16:54 by Johanna Nyborg Bendtsen Do you like this idea?

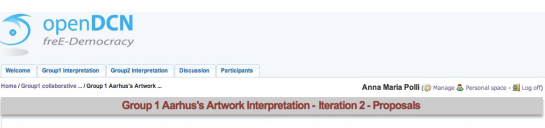

The scenario isn't in the proposal gathering phase. Wait for the end of this iteration to submit your ideas.

$\checkmark$ Iteration 1

Time - hours, minutes, seconds... Sometimes time flies by, and sometimes it feels so slow. We get older every second, and so does our things. "Waste not" is an exhibition with very near - you can literally see the time that has past in these things. In time stories are told. Happy, sad and tragic ones. These stories live on in the things we posses. What story
those your things tell? What is your story? And in time, how will people respond to your those your things tell? What is your story? And in time, how will people respond to your

Written the 2014-02-21 at 16:54 by Johanna Nyborg Bendtsen
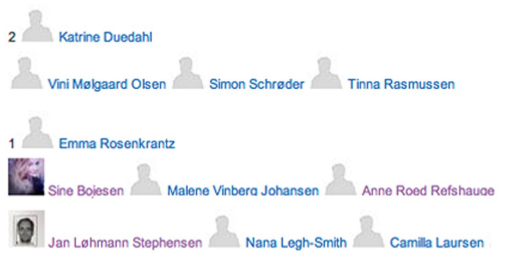

It is dominated by other ideas, so it is discarded.
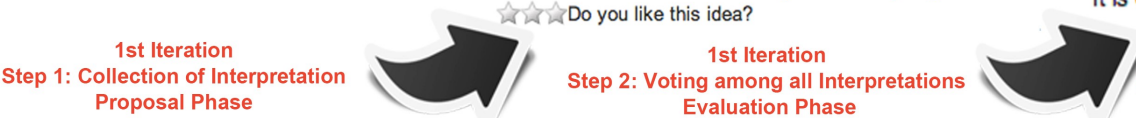

1st Iteration

Step 3: Results of Evaluation Interpretations Calculation Phase

Figure 2: Screenshots of the DCN platform - The transition from the first (collecting interpretations) to the second (voting on the interpretations) and the third phase (reading the evaluation) represents a full cycle of one iteration. 


\subsection{Step 3: Results of the evaluation (Calculation Phase)}

Upon completion of the evaluation by the HBGA, the highestscored interpretations were listed first and labeled highlighted (see Figure 2 - right side), and interpretations with lower rankings (low fitness) were labeled discarded and therefore listed below the interpretations that are labeled as highlighted. In the Calculation Phase, information about authorship and ranking of each interpretation was revealed. Subsequently, a new iteration started, and all participants had another chance to compose an interpretation by improving and rewriting the highlighted interpretations. The participants were asked to use the highestvoted interpretations as a basis for writing a new interpretation this is where the collaborative process started. The discarded interpretations were not considered further in the continuous writing process. Based on the highlighted interpretations, new interpretations were written (Step 1 - see above) and then another voting followed (Step 2 - see above). This iterative process was limited to a maximum of four cycles, by the end of which, we put to test whether the participants may arrive at a final interpretation that constituted the consensus of all highest-scored interpretations. If a consensus was reached, a collaborative interpretation of a specific artwork resulted.

\section{EVALUATION OF THE E-DELIBERATION PLATFORM}

\subsection{Research setting}

We designed the platform and evaluated it in a particular setting with the intent to understand the impact of the technology on the participants. When seeking to establish new practices, such as collaborative art interpretation writing, experiments need to be conducted first. The set-up of our e-Deliberation platform was put in place to address the tensions that emerged from the previous study $[4,25,33,34]$, which suggested continuing with the idea of collaborative writing, but in a different context, such as when being ex-situ (remote from the specified place (i.e., the art gallery). We sought to examine whether an e-Deliberation platform that supports collaborative writing could help to overcome these tensions. In our first study, we had the opportunity to conduct a study only for one exhibition period in the art gallery, which was one month. In contrast, this study was conducted for three months and we investigated how people engaged in writing interpretations, in particular those that came to see the exhibition only once and did not return.

\subsection{Participants' involvement}

With this study, we sought to intensify participants' involvement by providing activities that required using the platform at least twelve times in four iterations. Our new e-Deliberation platform was introduced and discussed in an introductory session (as explained in Section 5). All 23 participants attended the workshop, where they discussed the study procedure with the researchers. The participants registered and created personal accounts on the platform and familiarized themselves with it. This was a mandatory prerequisite for being able to interact with the platform. After the introductory workshop, the participants and one researcher visited an exhibition in the Kunsthal Aarhus art gallery in Denmark. The participants would write a collaborative interpretation of a specific artwork selected by the researchers, the students' lecturer and the staff of the art gallery. The piece of art consisted of a collection of 10.000 domestic objects, and was chosen with the participant group's age and interests in mind with the aim of them building a relationship and a connection to it. After the participants had been introduced to the artwork and the platform, they were invited to start writing their collaborative interpretations ex-situ.

\subsection{Data collection and analysis}

On the introductory day, seven on-site semi-structured group interviews were conducted (23 participants in groups of three to four), each of which lasted 40 to 60 minutes. In total, we collected around 4 hours and 15 minutes of qualitative interview data on that day. These opening interviews focused on current practices in an exhibition when it comes to discussing and interpreting art, and on previous experiences with writing collaborative texts or drafting interpretations. During the process of collaborative writing, the participants were asked to submit a photo and a diary entry describing the situation they were in when writing their interpretations. We recorded the full history of all submitted interpretations, votings and evaluations on the platform to track the evolution of the 23 interpretations from the beginning to the final version containing the consensus of the best interpretations. At the end of the study, we organized a final discussion lasting about an hour to debrief the participants about their experiences with the platform and the results of collaborative writing. The project was finalized on that day when we selected the main contributors to the final written interpretation. Further, we conducted and recorded semi-structured interviews with each student, in total around 5 hours and 45 minutes. We also logged all user activity on the platform and tracked the full evolution of the submitted interpretations.

The first author and another researcher from the team conducted the bottom-up analysis. To this end, we first transcribed approximately 12 hours of collected interview data using the F5 audio-transcription software. Five major themes of interest emerged from the data analysis: ex-situ appropriation, appropriation of technology, appropriation of the collaboratively written interpretation, the collaborative writing process and the experiences with the e-Deliberation platform. We classified the experiences and empirical material of the transcribed data according to these 5 themes. To obtain a better overview, we used the F4 Analyze audio-transcription software. Both researchers performed these steps independently and then combined the results.

\section{TECHNOLOGY APPROPRIATION PRACTICES}

A frequent observation in ethnographic studies is that people do not 'play by the rules'. Instead, they adapt, adopt and shape technology to their needs in ways the designer did not envisage [2, $3,4,5]$. Keeping this in mind, we collected various kinds of data to investigate the phenomenon of appropriation. From our empirical material, we identified appropriation practices that occurred over the course of the project.

\subsection{Identifying appropriation practices}

\subsubsection{Initial familiarity with Microsoft Word when} drafting an interpretation of an artwork

As observed in the art gallery, one practice of the visitors, curators and artists is to communicate about and interpret artworks. We previously explored how these communication needs can be supported in-situ [4, 25, 33, 34], and in this study presented in this paper, we explored the ex-situ needs through the use of our eDeliberation platform. The interface for drafting their interpretations was designed to resemble those the participants 
were familiar with from social media, such as Facebook or personal blogs. From our interview data, the first appropriation practice we identified was that some participants used a preexisting tool from their everyday practice. To ease interaction, communication and practice, they used Microsoft Word to draft their interpretations. Despite its close resemblance to that on Facebook and the textarea in Microsoft Word, the users participating in our study did not appropriate our textarea field and, surprisingly, reached for Microsoft Word, which is unrelated to the platform. The following quotes highlight that Microsoft Word was used to ease the interaction with our newly introduced technology: "I guess it is a habit, actually. It felt like writing an essay, when we do it in class or in school. And I guess it was sort of the same, so I did it in Microsoft Word, because I am used to doing it like this, writing some sort of text in a word document" (P2, Paragraph 54). Further, Participant 6 rejected using our text area field: "I used Word. Maybe because I feel more familiar with it, but also because I think I don't know if your website has the spell check, because it is in English, and you want to write something where there aren't any spelling mistakes." (P6, Paragraph 34). As Participant 6 pointed out, she did not only use Microsoft Word because she was more familiar with it, but also because Microsoft Word provides a spell check that she preferred to use, as she was writing in English, which was not her mother tongue. In her personal life she was also using Word as a substitute for the textarea field on her blog: "I have a blog, too, where I write, and actually there, too, I go into Word if it's long; if it's two lines I do it in there [the blog]. And again it is to double-check that you have the right grammar and the right words." (P6, Paragraph 35). About her interaction with the eDeliberation platform she said, "I wrote the interpretation always first in Word. [...] I wrote it in a Word document and then copypasted it into the box [of our platform].” (P6, Paragraph 250, 264) Participant 8 strengthened the previous statement by saying, "I used your platform just to upload my text. I wrote my text in another program, and then I just copied it and then uploaded it in your platform and submitted it there." (P8, Paragraph 8) The participants' arguments were that using a tool with which they were more familiar felt more comfortable when communicating their ideas about the interpretation of the piece of art than using the textarea field provided on the e-Deliberation platform. For instance, a participant said, "In [Microsoft] Word, I can easily edit and rewrite sections of my interpretation, save the draft of it, and change it later on maybe again before I copy, paste and submit it on the e-Deliberation platform." (P13, Paragraph 85)

In our analysis of the interview data, we found that many participants had stated that they had used Microsoft Word rather than our textarea field. The interface design of the e-Deliberation platform had the same features and functions for writing, rewriting or editing text. However, the main reason for the nonappropriation [23] of our textarea field was that the participants were more familiar with Word, and they preferred to have spell checking and saving functionalities available, and they did not test whether our software supported these. Since they were accustomed to creating longer texts in Microsoft Word, they used it to draft their interpretations, and when they were satisfied with the interpretation they had written, they would copy the whole text, paste it into our textarea field and submit the interpretation. Another reason for using Word as substitute software was that "it was difficult to find the place where you had to write your interpretation in, especially after the first one. When you have to edit it, then you have to find your own interpretation and you sometimes had to write a new one. It was not that easy. The system could be user-friendlier." (P10, Paragraph 12) To Participant 10, the interface was unclear, and therefore he did not appropriate our platform tool, but participated in the following cycles: "I still wanted to vote, that was okay, but to write and edit my interpretation - that was difficult" (P10, Paragraph 21). Participant 10 continued with some tasks on our platform, but declined to continue writing collaborative interpretations.

In the subsequent steps of the collaborative writing process, the eDeliberation platform was used to support reading and evaluating each other's submitted interpretations and to brainstorm and create a new interpretation based on the highlighted submissions. When the next iteration of the writing process started, some students chose again to use Microsoft Word for drafting their interpretations and including parts from the other interpretations submitted. Even though a few participants pointed out that the interface had similarities to Facebook, where "one would just post a comment or an idea in a small textbox" (P8, Paragraph 41), the interpretations were not written directly in the textarea field on the e-Deliberation platform, but in Microsoft Word. These improvisations and adaptations around our online technology to communicate and better convey their ideas show the technology has been domesticated. The participants used the technology in their own ways and not simply as we - the designers - had intended.

We have previously investigated the potential of previous practices to affect early use and its further development [4]. One can argue that initial familiarity with technology use based on everyday practices is an influential factor that might guide the use of a new technology [2]. In this case, we found that appropriation of the technology might be affected by initial familiarity based on everyday practices. When a technology is used in everyday life for a recurring purpose, then initial familiarity may influence how a novel technology will be used, which can lead to unexpected appropriation practices. This points towards an involvement of different kinds of past experience that were not anticipated by the designer [4].

\subsubsection{Gaining a sense of ownership}

We also identified a second appropriation practice, namely gaining a sense of ownership, categorized by Alan Dix as an advantage of technology appropriation [3]. The participants felt more in control when completing their tasks in their own way. They stated that, over the three-month period of frequently using the e-Deliberation, they had formed a strong connection to the technology and their written interpretations. Because of their frequent use of the technology, participants were able to bring their own ideas and personal thoughts into their written interpretations and integrate other ideas into their own interpretations.

At the end of the study, a consensus among the final submissions of the interpretations was reached. One participant even compared our platform to Wikipedia, as she thought that our e-Deliberation platform worked exactly like the free online encyclopedia, which is created and edited by volunteers. This participant stated, "In the start of the study my thoughts upon the project were, we would all do a Wikipedia text. [...] I really thought that it should end up like this one text, and then we would all go edit and rewrite the text, like in Wikipedia." (P2, Paragraph 12,313)

After agreeing on one from the 23 as the final interpretation, one participant achieved the highest final student evaluations and was therefore adjudged to have written the interpretation that best synthesized all of the submissions. When the winner was announced to the other participants, one student pointed out, "I 
know that the winner had actually copied directly from my former interpretation, and I think that wasn't really the job. I didn't copy anything; I maybe have copied an idea and formulated it in my own words [...], and I think that is kind of unfair if the first six lines are actually from another person." (P6, Paragraph 19, 20) Another participant stated, "There were some parts that were similar in all the texts, so in that way there was some of mine in it as well." (P11, Paragraph 78) As these quotes demonstrate, some participants developed a sense of ownership of their public interpretations through the web-based technology. Their perceived ownership of these interpretations was well developed, although writing a collaborative interpretation involved some ideas from their colleagues that were discarded at the end of the process. In the interviews, we observed how participants considered their written interpretations to be their own. They identified them as their private property and took offence when someone else copied parts of their interpretations into their own.

\subsection{Appropriation related to communication}

In museums, one can observe groups of visitors interested in art discussing and interpreting together the pieces on show. When in a group, people can communicate with each other about a piece of art in-situ. However, they lack this opportunity when wandering through an exhibition on their own [35]. This is one of the main reasons why we deployed this technology, so visitors can leave a trace behind them by expressing their impressions of an artwork. Visitors to the museum can then read and comment on written statements by past visitors. Thus, this platform was created to enable visitors to communicate with each other without necessarily being physically present in the art gallery, which creates space for interaction and for expressing different ideas.

In particular, previous visitors' comments on a piece of art inspire new visitors to see it from a completely new perspective. A participant expressed, "It is interesting to see how other people interpret the piece, or having some thoughts that I never thought of when I saw the artwork. " (P1, Paragraph 53) Similarly, another participant stated, "It was nice because you get another point of view on the artwork. So the things that I felt I could use and took for my own interpretation, and the things I didn't really see or agreed with I didn't." (P2, Paragraph 90) These feelings are more likely to arise when in a group that enables people to discuss a piece of art in-situ. "I often go with my fellow art students and we usually talk a lot about it, and again I think it is very intuitive, the colors or the forms that attract me, and then I look at the picture. I don't stop at every picture and read, and how I feel again. If I have a comment I tell it to my girl friends. " (P6, Paragraph 175) The e-Deliberation platform technology addresses these communication needs. It also allows people to express and communicate their ideas and thoughts. For example, a participant commented, "I feel a stronger connection to the artwork than I would if I just read a text beside the artwork that some person in a museum had made. Like I feel a stronger connection to the artwork; like I remember it better, and I like talking about it as well. I like discussing it with other people. (P2, Paragraph 220). Another participant said, "Of course it is about interpreting art and not just standing there and not talking about it. It is about to get people to write and to talk about it; that is a success, I think. Because when I go to a museum, I don't talk so much afterwards. It is just when I am actually there or when I am on the way home I'll talk about it. But this new way is maybe a better way." (P3, Paragraph 16)

Our analysis of the interviews also revealed unexpected communication needs from the platform. In the interviews at the end of the study, participants stated that they needed to talk about and discuss face-to-face how to use the e-Deliberation platform. For example, one stated, "In the middle of the project I got confused [...], but then we talked about it a lot, and that helped." (P2, Paragraph 20). Furthermore, asked with whom they were talking about the platform, they said, "with my class mates, that we could ask questions, as it was a bit confusing. I don't know why; like, in the start I really had a good feeling what your project was about, and then, maybe because we were discussing a lot in the class as well, and then maybe just developed into something else." (P2, Paragraph 23)

More interestingly, the participants discussed how to read interpretations, how to phrase their ideas and what to include from the other interpretations submitted. We asked them what form these discussions in class took, and they stated, "It was, like, in the hallways." (P2, Paragraph 25) They discussed face-to-face: "[...] like, what did you write? And what are your thoughts of this?" (P2, Paragraph 25) These kinds of communication needs were completely unexpected from the designers' perspective.

In summary, we identified two communication needs in our study. These can be characterized as the need to talk about (i) the content of the collaborative writing process and (ii) use of the new technology. Although tools for both needs were available on the e-Deliberation platform, they were not used. Either the participants did not discover the capabilities of the technology, or the technology failed while showing its capabilities. As Carroll would characterize it, this tool was non-appropriated [23].

\subsection{Embedding Practices and Needs in Context}

We explored two different contexts: one in which the participants were invited to write an interpretation in-situ while close to the artwork and the second ex-situ, when the users wrote an interpretation remotely. In-situ, the participants appropriated the technology differently than anticipated and used the technology to write or leave some notes like they would write in a guestbook or to write Facebook-style comments, etc. These unexpected uses militated against achieving one final coherent collaboratively written text (details of the appropriation practices in-situ can be found in [4]). However, in the ex-situ case, the participants domesticated the technology in various places around their homes. Asked in which situations they felt comfortable using the technology and writing their interpretation, the participants pointed out, "I was sitting on my couch with my dog next to me and writing the interpretation," (P9, Paragraph 114) or, "When I have to write a longer text, I like to sit upright in my bed. There I have time to think in a comfortable environment." (P13, Paragraph 116) The participants used the technology mainly at home, either while sitting on a couch or at their kitchen tables, or relaxing in their beds. When participants used the technology exsitu, they had significantly more time to reflect upon the artwork's meaning and were therefore able to write a coherent collaborative interpretation rather than only a short comment like the users in the in-situ project.

\section{DISCUSSION}

\subsection{Deriving an informed basis for research and design}

Designing for appropriation is often seen as an oxymoron, as it seems difficult to design for the unexpected [3]. While much has been written about the importance of appropriation $[1,2,5]$, it is 
difficult to find explicit design guidelines or practical advice on how to design for appropriation [3,6]. As Dix and Carroll wrote: "design can never be completed, but you can design to allow the unexpected" $[2,3]$, and one can learn from it for the next design iteration. The analytical method applied and presented helps in drawing attention to the specific opportunities for, and obstacles to, appropriation in our context. Our analysis identified two points in which our e-Deliberation platform constrained appropriation and one in which it enabled successful appropriation.

\subsubsection{Implications of our findings for the development of the e-Platform}

Our e-Deliberation platform gave rise to two challenges regarding appropriation of e-Applications. Our preliminary analysis showed that pre-existing everyday practices and initial familiarity have a pivotal influence on how a new technology will be appropriated. What the users of an e-Application usually interact or work with influences usage and future appropriation. However, at this stage it is difficult to make assumptions about which existing practices from past experiences users will apply when they recognize a design on the basis of initial familiarity and when thinking about how a new design might be used in the future. This phenomenon is examined in more detail below.

The first cause of non-appropriation of the platform resulted from rejecting one specific tool: the users of our platform preferred to use an unrelated tool with which they were familiar from their everyday lives and practices. In particular, they used Microsoft Word to draft their interpretations (since they knew it from their everyday practices) rather than the 'textarea field to write interpretations in' tool. Non-appropriation of this one tool neither hindered nor interrupted them in participating in the collaborative writing process. However, it shows that, when users are not comfortable using a tool of an e-Deliberative platform, they nonappropriate or may even dis-appropriate the technology. Therefore, we recommend that the design of an e-Application be based on features that users are instantly familiar with and use in their everyday lives to complete related tasks. Nonetheless, even then non-appropriation cannot be prevented. Our study has shown (see Section 7.1.1) that non-appropriation occurs also in their daily use of e-Applications (e.g., writing for their personal blogs). Non-appropriation of a novel technology may be the result of nonappropriation of a tool they use in their everyday lives. Being used to replacing the existing tool on their blog with Microsoft Word influenced their use of our e-Deliberation platform. Once a tool has been non-appropriated and replaced by another, this will influence the appropriation of similar tools in other eApplications. Once non-appropriated, there is also a high chance a tool will remain non-appropriated in new e-Applications. Both aspects are therefore important: what tools users are already familiar with and what tools they have declined to appropriate in their everyday lives.

We designed an installation with strict regulations, meaning there was little initial learning to be expected. Our study shows that, nonetheless, users bring to the table past experiences that strongly influence the likelihood of future appropriation. Some participants returned to using a familiar tool to complete certain tasks. If there had been other tools from their daily use that participants could have gone back to and replace the new ones with, would they have done so? As Dix described, there may be a method or a tool to fulfill a task within the newly designed technology, but sometimes non-appropriation of a provided feature is easier - at that particular moment or because it eliminates the learning effort involved [3]. Dix's example illustrates, similarly as ours, that "using emails for sharing files instead of configuring a shared network folder" is one way to appropriate an e-Application because it is easier and practical at that particular moment. In our case, the participants were not willing to learn how to use a new technology for a certain task when it was easier to reach for another tool with which they were already familiar. The use of Word has shown that participants would rather use a tool with which they are initially familiar than use a new tool designed specifically to complete the task.

Our initial experimental design assumption was that the eDeliberation platform would meet users' communication needs within the constraints defined. Despite our attempt to anticipate the unexpected in design terms, our preliminary analyses appear to indicate that this formal restriction results in 'unsuccessful' appropriation - the second problem. As shown by the quotes in Section 7.2, a lack of knowledge about and lack of practice with the newly introduced e-Deliberation platform and the lack of space for communication gave rise to 'unsuccessful' appropriation. When looking at users' appropriation behaviors, we identified the following communication needs that the eDeliberation platform obviously did not support. Although we provided a tool for communication and discussion, the participants turned as a group to face-to-face communication to raise and clarify questions relating to the e-Platform, the process of the project, and how to draft an interpretation. Here the communication needs were not satisfied due to 'unsuccessful' appropriation of the e-Deliberation platform. This 'unsuccessful' appropriation resulted in users talking face-to-face to discuss the artwork instead of using the e-Deliberation platform, which is a sign of non-appropriation in the process of the collaborative writing. In this particular case, face-to-face communication was easy because the students were attending the same course on a weekly basis, where open questions could be addressed. In an eApplication or research project that lacked this opportunity, users would most likely dis-appropriate the e-Application on encountering the first problems.

These two causes of non-appropriation can be removed, firstly by providing a design they are familiar with from their everyday lives and secondly by implementing a discussion forum that is easy to use and does not require an initial learning phase.

\subsection{The design of future e-Deliberation platforms}

The third example of appropriation practice is related to the "sense of ownership" which occurred after frequent use of our eDeliberation platform, when participants acquired a feeling of control. Allowing the user to gain a sense of ownership is key to the design of our e-Deliberation platform, as it sustains participation by enabling users to shape, adopt and adapt the interpretations, as they desire, as presented in Section 7.1.2. Remarkably, through the deployment of the e-Deliberation platform ex-situ and applying the human-based genetic algorithm, participation was sustained throughout all iterative cycles, and one final consensus on one collaborative interpretation was reached. In our previous research case, we did not succeed in these aspects, as our system enabled collaborative interpretation only on site [25]. The participants in the study presented here had no problems picturing themselves taking part in the activity of writing collaboratively an interpretation, which would then hang in the gallery beside the artwork.

Our goal was to enable the kind of location agnostic collaborative deliberation that is provided by Wikipedia. Interestingly, our 
participants even compared our platform to Wikipedia, as they expected it to work in the same way (as presented in Section 7.1.2). However, we observed that the participants used the system primarily to vote on and evaluate the last few submissions, rather than to compose continuous interpretations - a phenomenon also observed when the openDCN was used in Milan to foster public discussions and a sense of community [27]. However, we argue that this process of participation is key to achieving one contextual consensus of the submitted interpretations, leading to one final written interpretation emerging from applying the human-based genetic algorithm.

\section{CONCLUSION}

We have presented an ex-situ e-Deliberation platform that uses a human-based genetic algorithm and collaboratively written interpretations as main components. We have demonstrated how our platform works in the context of e-Deliberation to support citizen involvement through the development of a collaboratively written interpretation. Given the positive feedback from the participants in our study, we consider such a platform an excellent opportunity for art galleries or similar institutions to connect more strongly to their visitors.

The main contributions of this paper are the representations [36] of unexpected practices and appropriation experiences using an eDeliberation platform that imposes strict regulations and can be useful for cooperative design, as it can facilitate an explicit understanding of a collaborative writing situation at hand. We have presented two hindrances that constrain and one benefit that promotes appropriation, which can inform the process of design to maximize appropriation. These findings can serve as an example for other collaborative e-Applications, and this design recommendation plays a large role for e-Applications for successful appropriation. Further, on the basis of these findings, we plan to extend our work by informing a hybrid of in- and exsitu applications that sustain participation by removing barriers and applying enablers supporting technology appropriation. Our study has some limitations, as it involved only a relatively small number of participants; consequently, our findings should be considered qualitative and indicative.

\section{ACKNOWLEDGMENTS}

We thank our collaborators in the project: from the Aarhus University Susanne Bødker, Clemens Nylandsted Klokmose, Matthias Korn, Jan Løhnmann, researchers from our previous study and the participants. From the Milan University, we want to thank Fiorella De Cindio, Leonardo Sonnante and the programmers of the e-Deliberation platform technology. The evaluation is based on a research work, which uses a quite sophisticated algorithm, proposed by Pietro Speroni di Fenizio and Derek Paterson from University of Milan. This research has been funded by Aarhus University's interdisciplinary research center Participatory IT, PIT.

\section{REFERENCES}

[1] P. Dourish, "The appropriation of interactive technologies: Some lessons from placeless documents," Comput. Support. Coop. Work CSCW, vol. 12, no. 4, pp. 465-490, 2003.

[2] J. Carroll, S. Howard, F. Vetere, J. Peck, and J. Murphy, "Just what do the youth of today want? Technology appropriation by young people," in System Sciences, 2002. HICSS. Proceedings of the 35th Annual Hawaii International Conference on, 2002, pp. 1777-1785.
[3] A. Dix, "Designing for appropriation," in Proceedings of the 21st British HCI Group Annual Conference on People and Computers: HCI... but not as we know it-Volume 2, 2007, pp. 27-30.

[4] S. Bødker and A. M. Polli, "Between Initial Familiarity and Future Use: A Case of Collocated Collaborative Writing," in COOP 2014 - Proceedings of the 11th International Conference on the Design of Cooperative Systems, 27-30 May 2014, Nice (France), C. Rossitto, L. Ciolfi, D. Martin, and B. Conein, Eds. Springer International Publishing, 2014, pp. 137-154.

[5] E. Grönvall and N. Verdezoto, "Beyond Self-monitoring: Understanding Non-functional Aspects of Home-based Healthcare Technology," in Proceedings of the 2013 ACM International Joint Conference on Pervasive and Ubiquitous Computing, New York, NY, USA, 2013, pp. 587-596.

[6] A. M. Al Zubaidi-Polli, "Comparative Study of Technology Appropriation Concepts," in Proceedings of IEEE Student Branch Conference Mikulov 2017, 2017, pp. 3-6.

[7] G. Kotsis and I. K. Ibrahim, "The Web Goes Mobile: Can we Keep the Pace?," in 2008 International Conference on Complex, Intelligent and Software Intensive Systems, 2008, pp. 240-246.

[8] S. Draxler and G. Stevens, "Supporting the Collaborative Appropriation of an Open Software Ecosystem," Comput. Support. Coop. Work CSCW, vol. 20, no. 4-5, pp. 403448, Oct. 2011.

[9] S. L. Bryant, A. Forte, and A. Bruckman, "Becoming Wikipedian: transformation of participation in a collaborative online encyclopedia," in Proceedings of the 2005 international ACM SIGGROUP conference on Supporting group work, New York, NY, USA, 2005, pp. $1-10$.

[10] D. Johansson, J. Lassinantti, and M. Wiberg, "Mobile eServices and Open Data in e-Government Processes: Transforming Citizen Involvement," in Proceedings of the 17th International Conference on Information Integration and Web-based Applications \& Services, New York, NY, USA, 2015, p. 8:1-8:10.

[11] C. Strauss, G. Kotsis, E. Pardede, and F. Xhafa, "Special issue on challenges with defining and measuring e-Services sustainability: Editorial preface.," J. Serv. Sci. Res. Heidelb. 42 Dec 2012, vol. 4, no. 2, pp. 169-173, 2012.

[12] M. Bohøj, N. G. Borchorst, S. Bødker, M. Korn, and P.-O. Zander, "Public Deliberation in Municipal Planning: Supporting Action and Reflection with Mobile Technology," in Proceedings of the 5th International Conference on Communities and Technologies, New York, NY, USA, 2011, pp. 88-97.

[13] J. Preece and B. Shneiderman, "The Reader-to-Leader Framework: Motivating Technology-Mediated Social Participation," AIS Trans. Hum.-Comput. Interact., vol. 1, no. 1, pp. 13-32, Mar. 2009.

[14] P. Baglietto, M. Fornasa, M. Maresca, and M. Stecca, "Collaboration and Real-time Analysis in the Spreadsheet Space," in Proceedings of the 17th International Conference on Information Integration and Web-based Applications \& Services, New York, NY, USA, 2015, p. 37:1-37:6.

[15] S. Bødker, P. Ehn, J. Knudsen, M. Kyng, and K. Madsen, "Computer Support for Cooperative Design (Invited Paper)," in Proceedings of the 1988 ACM Conference on 
Computer-supported Cooperative Work, New York, NY, USA, 1988, pp. 377-394.

[16] O. S. Iversen and R. C. Smith, "Connecting to Everyday Practices: Experiences from the Digital Natives Exhibition," in Heritage and Social Media, Routledge / Lawrence Erlbaum Associates Publ, 2012.

[17] S. Bødker, C. N. Klokmose, M. Korn, and A. M. Polli, "Participatory IT in Semi-public Spaces," in Proceedings of the 8th Nordic Conference on Human-Computer Interaction: Fun, Fast, Foundational, New York, NY, USA, 2014, pp. 765-774.

[18] E. Balka and I. Wagner, "Making Things Work: Dimensions of Configurability As Appropriation Work," in Proceedings of the 2006 20th Anniversary Conference on Computer Supported Cooperative Work, New York, NY, USA, 2006, pp. 229-238.

[19] G. DeSanctis and M. S. Poole, "Capturing the Complexity in Advanced Technology Use: Adaptive Structuring Theory," presented at the Organ. Sci., 1994, vol. 5, pp. 121-147.

[20] W. March, M. Jacobs, and T. Salvador, "Designing Technology for Community Appropriation," in CHI '05 Extended Abstracts on Human Factors in Computing Systems, New York, NY, USA, 2005, pp. 2126-2127.

[21] P.-A. Quinones, S. D. Teasley, and S. Lonn, "Appropriation by Unanticipated Users: Looking Beyond Design Intent and Expected Use," in Proceedings of the 2013 Conference on Computer Supported Cooperative Work, New York, NY, USA, 2013, pp. 1515-1526.

[22] C. Bossen and P. Dalsgaard, "Conceptualization and appropriation: the evolving use of a collaborative knowledge management system.," 2005, pp. 99-108.

[23] J. Carroll, "Completing Design in Use: Closing the Appropriation Cycle," ECIS 2004 Proc., Jan. 2004.

[24] J. C. Jones, "Continuous design and redesign," Des. Stud., vol. 4, no. 1, pp. 53-60, Jan. 1983.

[25] A. M. Polli, M. Korn, and C. Nylandsted Klokmose, "Local Area Artworks: Collaborative Art Interpretation On-site," in Proceedings of the 2013 ACM Conference on Pervasive and Ubiquitous Computing Adjunct Publication, New York, NY, USA, 2013, pp. 79-82.
[26] F. De Cindio, "Guidelines for Designing Deliberative Digital Habitats: Learning from e-Participation for Open Data Initiatives," J. Community Inform., vol. 8, no. 2, Apr. 2012.

[27] F. De Cindio, A. De Marco, and L. A. Ripamonti, "Enriching Community Networks by supporting deliberation," in Communities and Technologies 2007, Springer, 2007, pp. 395-417.

[28] F. Di Cindio and C. Peraboni, "Internet as a platform for political engagement: from protests to proposals," in Proceeding of the CIRN-DIAC community informatics conference, 2010.

[29] P. S. di Fenizio and D. Paterson, "Don't Vote, Evolve!," in Electronic Participation, 2010, pp. 13-25.

[30] P. S. Di Fenizio and C. Anderson, "Using Pareto Front for a Consensus Building, Human Based, Genetic Algorithm," in Proceedings of the 10th European Conference on Advances in Artificial Life: Darwin Meets Von Neumann Volume Part II, Berlin, Heidelberg, 2011, pp. 175-182.

[31] A. Kosorukoff, "Human based genetic algorithm," in 2001 IEEE International Conference on Systems, Man and Cybernetics. e-Systems and e-Man for Cybernetics in Cyberspace (Cat.No.01CH37236), 2001, vol. 5, pp. 34643469 vol.5.

[32] A. M. Al Zubaidi-Polli and N. Verdezoto, "Collaborative Technology Appropriation: A Case of Groups Using a Deliberative Community Network," In Preparation.

[33] F. Di Cindio and C. Peraboni, "Fostering eParticipation at the Urban Level | Technology | World Wide Web," Springer-Verl. Berl. Heidelb. 2009.

[34] M. Korn, A. M. Polli, and C. N. Klokmose, "Experiences from a Real-Time Mobile Collaborative Writing System in an Art Gallery," in International Workshop on Designing Mobile Face-to-Face Group Interactions, Paphos, Cypern, 2013.

[35] N. Simon, The Participatory Museum, First Edition. Santa Cruz, Calif.: Museum 2.0, 2010.

[36] M. Kyng, "Making Representations Work," Commun $A C M$, vol. 38, no. 9, pp. 46-55, Sep. 1995. 